\title{
Multiple-hook fixation in revision spinal deformity surgery for patients with a previous multilevel fusion mass: technical note and preliminary outcomes
}

\author{
Ning Liu, MD, ${ }^{1,3}$ and Kirkham B. Wood, MD ${ }^{1,2}$ \\ ${ }^{1}$ Department of Orthopaedic Surgery, Spine Service, Massachusetts General Hospital, Boston, Massachusetts; ${ }^{2}$ Department of \\ Orthopaedic Surgery, Stanford University Medical Center, Redwood City, California; and ${ }^{3}$ Washington University in St. Louis, \\ Brown School, St. Louis, Missouri
}

OBJECTIVE A previous multilevel fusion mass encountered during revision spinal deformity surgery may obscure anatomical landmarks, making instrumentation unworkable or incurring substantial blood loss and operative time. This study introduced a surgical technique of multiple-hook fixation for fixating previous multilevel fusion masses in revision spinal deformity surgeries and then evaluated its outcomes.

METHODS Patients with a previous multilevel fusion mass who underwent revision corrective surgery down to the lumbosacral junction were retrospectively studied. Multiple hooks were used to fixate the fusion mass and linked to distal pedicle screws in the lumbosacral-pelvic complex. Radiological and clinical outcomes were evaluated.

RESULTS The charts of 8 consecutive patients with spinal deformity were retrospectively reviewed (7 women, 1 man; mean age 56 years). The primary diagnoses included flat-back deformity (6 cases), thoracolumbar kyphoscoliosis (1 case), and lumbar spondylosis secondary to a previous scoliosis fusion (1 case). The mean follow-up duration was 30.1 months. Operations were performed at T3/4-ilium (4 cases), T7-ilium (1 case), T6-S1 (1 case), T12-S1 (1 case), and T9-L5 (1 case). Of 8 patients, 7 had sagittal imbalance preoperatively, and their mean C-7 plumb line improved from $10.8 \pm 2.9 \mathrm{~cm}$ preoperatively to $5.3 \pm 3.6 \mathrm{~cm}$ at final follow-up $(p=0.003)$. The mean lumbar lordosis of these patients at final follow-up was significantly greater than that preoperatively $\left(35.2^{\circ} \pm 12.6^{\circ}\right.$ vs $16.8^{\circ} \pm 11.8^{\circ}$, respectively; $\left.p=0.005\right)$. Two perioperative complications included osteotomy-related leg weakness in 1 patient and a stitch abscess in another.

CONCLUSIONS The multiple-hook technique provides a viable alternative option for fixating a previous multilevel fusion mass in revision spinal deformity surgery.

https://thejns.org/doi/abs/10.3171/2016.8.SPINE16432

KEY WORDS multiple-hook fixation; spinal deformity; revision surgery

$\mathrm{R}$ EVISION spinal deformity surgery is often complicated by a posterior fusion mass from previous surgeries. Thoracolumbar long fusions have the potential to promote distal adjacent-segment degeneration and overall spinal alignment deterioration that may eventually warrant surgical revision, especially in older patients whose compensatory mechanisms are less effective., ${ }^{3,10}$ This phenomenon is often associated with previous posterior nonsegmental thoracolumbar distraction instrumentation. ${ }^{10}$

Uncommonly but not rarely, some of these fusion masses span multiple levels, and they pose a challenge for spine surgeons. Placement of pedicle screws in such regions is extremely difficult, because the anatomical landmarks are thoroughly obscured. It may be possible to find the pedicle screw tracts, but it would invite the generous removal of fused bone and exhaustive dissection, with substantial operative time and blood loss. One alternative would be using a computer-aided navigation system to guide pedicle screw placement, but such equipment is not widely available and not every surgeon is familiar with these tools. Another option is to leave the previous fusion mass intact and to bridge the instrumentations at their proximal and distal adjacent ends, but this is usually not feasible because excluding long fusion masses from fixation would significantly increase the risk of instrumentation failure. ${ }^{5}$

We have used multiple laminar hooks to fixate these multilevel fusion masses in revision spinal deformity surgeries by making anchoring sites directly on the fusion

ABBREVIATIONS EBL = estimated blood loss; PSO = pedicle subtraction osteotomy.

SUBMITTED April 20, 2016. ACCEPTED August 31, 2016.

INCLUDE WHEN CITING Published online December 9, 2016; DOI: 10.3171/2016.8.SPINE16432. 
mass. This method offers a technically simple and less invasive way to fixate the massive fusion mass. This retrospective review reports the preliminary radiological and clinical outcomes of this technique in 8 patients who were operated on by 1 senior surgeon (K.B.W.).

\section{Methods \\ Study Sample}

Following an exemption from the institutional review board, the charts of 8 consecutive patients with spinal deformity and a previous massive fusion mass, who underwent revision surgeries to the lumbosacral junction using the multiple-hook technique between November 2006 and April 2014, were studied. The indications for this technique included: 1) the inability to identify pedicle screw tracts and other anatomical landmarks due to a previous massive fusion mass that spanned 3 or more levels; and 2) an adequate thickness (usually $2 \mathrm{~cm}$ and above) of the fusion mass for hook placement, which was confirmed preoperatively by CT sagittal reconstruction.

Before discharge, patients were advised to visit our clinic at 3 months, 6 months, 1 year, and 2 years postoper- atively. The preoperative and follow-up workups included an entire standing lateral spine radiograph with the knees and hips fully extended.

\section{Operative Procedure}

In the prone position, a longitudinal incision was made incorporating the old incision. Hook placement on the fusion mass and pedicle screw placement on the other segments was performed before decompression or osteotomy. The hooks were placed in pairs, no less than $1.5 \mathrm{~cm}$ apart (to avoid "peeling off" the fusion mass) and facing each other, to form a clawlike structure. Such claw formations were placed onto the fusion mass on both sides, and they were staggered bilaterally to evenly hold the fusion mass. The number of hooks used depended on the length of the fusion mass, and we intrinsically followed the general principle of hook instrumentation for kyphosis with multiple claw configurations on each side. First, a steel bur with a medium-sized ball tip was used to perforate the surface of the fusion mass to gently enlarge and deepen the entry hole (Fig. 1A). Then, an angled curette or a laminar finder was used to dig a horizontal tunnel from the base of the entry hole to accommodate the incoming hook (Fig. 1B).
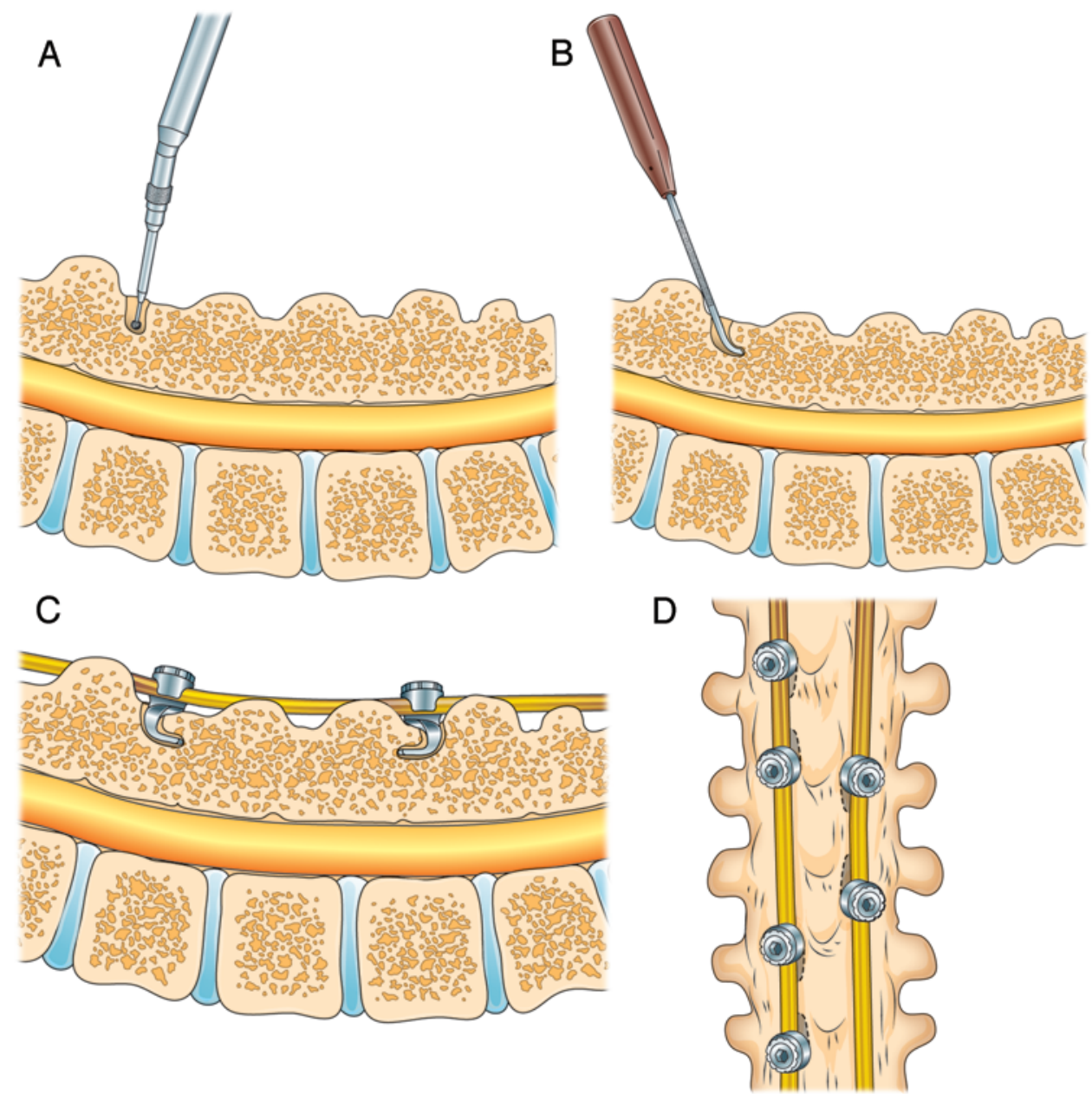

FIG. 1. Illustration showing the operative procedure. A: Burring the placement site for a hook on the fusion mass. B: Preparing a tunnel to accommodate the hook. C and D: The claw formation is made up of two hooks facing toward each other. Copyright Ning Liu. Published with permission. Figure is available in color online only. 
Care was taken to avoid entering the spinal canal below. After the anchoring points were ready, the hooks were placed in claw formations and were eventually connected with rods (Figs. 1C, 1D, and 2). A compressive force was applied to each claw formation before the nuts were tightened. It should be noted that the hook anchoring sites were prepared where the fusion mass was adequately thick and robust, and 1 single hook can be placed as needed in addition to the claw constructs.

The other steps of the operation including pedicle screw placement, decompression, and osteotomy were performed in the conventional manner. Five of 8 patients underwent anterior-posterior combined surgery. Five patients underwent osteotomies. In 6 patients, there was at least 1 segment of pedicle screws above and below the end hooks on the fusion mass, whereas in the other 2 (Cases 6 and 8), the proximal hooks on the previous fusion mass served as the upper end implant. Four patients had previous posterior implants. In 2 patients (Cases 1 and 8), we did not remove the Harrington rod; instead, we connected it to the newly placed rods with wedding-band connectors. Cross-links and double-rod structures were used as needed to strengthen the construct. Fusion was performed using autologous bone graft from the surgical site or ilium harvest.

\section{Evaluation of Surgical Outcomes}

Surgical outcomes were investigated radiologically and clinically. Four radiological parameters were measured and compared between intervals (Fig. 3). Thoracic kyphosis was defined as the Cobb angle between the upper endplate of T-5 and lower endplate of T-12. Lumbar lordosis was measured between the upper endplates of T-12 and $\mathrm{S}-1$. The pelvic tilt was the angle between the vertical line and the line joining the middle of the sacral plate and the center of the femoral head. For the C-7 sagittal plumb line, the posterosuperior aspect of the S-1 body was used as a reference point, and $0-4 \mathrm{~cm}$ from this point was considered to be within the normal range. ${ }^{1}$

Clinical outcomes included the operative time, estimated blood loss (EBL), perioperative complications, and follow-up outcomes that were evaluated by reviewing the clinic notes before surgery and at follow-up examinations. The follow-up outcomes were rated using a modified Pheasant 3-point scale: 1) favorable, all-sided improvement in back pain, posture, and activities of daily living; 2) fair, partial improvement, and the patient expressed satisfaction; and 3) unfavorable, partial improvement, and the patient expressed disappointment, no improvement, or deterioration. ${ }^{8}$

\section{Statistical Analysis}

A paired-sample t-test or rank-sum test was used to compare pre- and postoperative lumbar lordosis and the C-7 plumb line. SPSS software (version 13.0, SPSS Inc.) was used for data analysis, and the $\alpha$ value was set at 0.05 .

\section{Results}

\section{Patient Demographics}

Eight patients were examined ( 7 women and 1 man) with an average age of 56 years (range 50-69 years). The mean follow-up duration was 30.1 months (range 4-90 months) (Table 1). Six patients were diagnosed with flatback syndrome, 1 had thoracolumbar kyphoscoliosis, and 1 had lower lumbar adjacent-segment disc disease due to a previous Harrington rod surgery for scoliosis. All patients had significant back pain or a posture problem that was refractory to nonoperative treatment. Of the 8 patients, 7
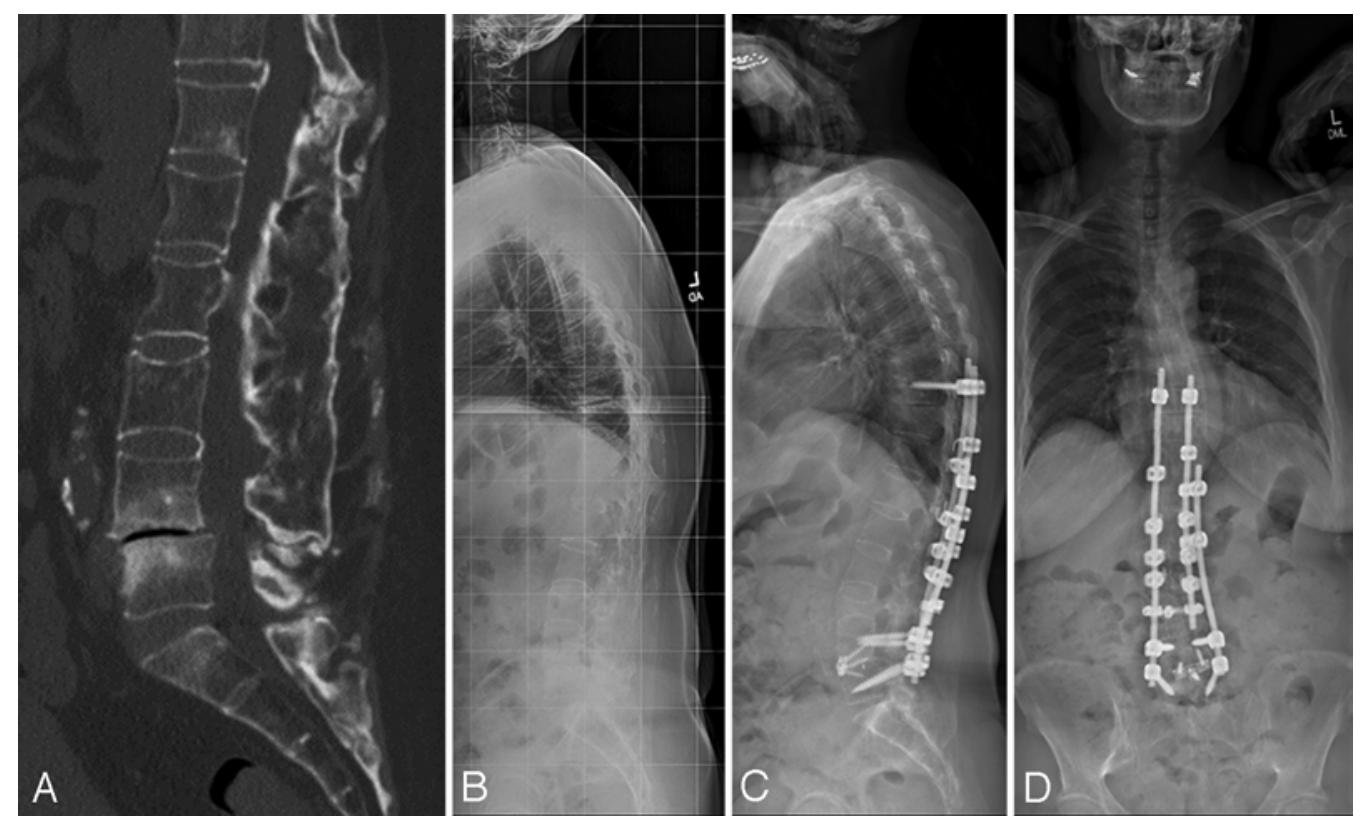

FIG. 2. Case 5. Preoperative CT image (A) and radiograph (B) of patient who was diagnosed with a flat-back deformity show the massive fusion mass from a previous spinal fusion in 1978. She underwent an anterior-posterior combined corrective surgery wherein multiple hooks were used to fixate the previous fusion mass between T-10 and L-2. On radiographs obtained 30 months postoperatively (C and D), her C-7 plumb line was $3.8 \mathrm{~cm}$ (preoperatively $9.1 \mathrm{~cm}$ ), and the corresponding clinic note showed that she was "very happy that she had surgery." 

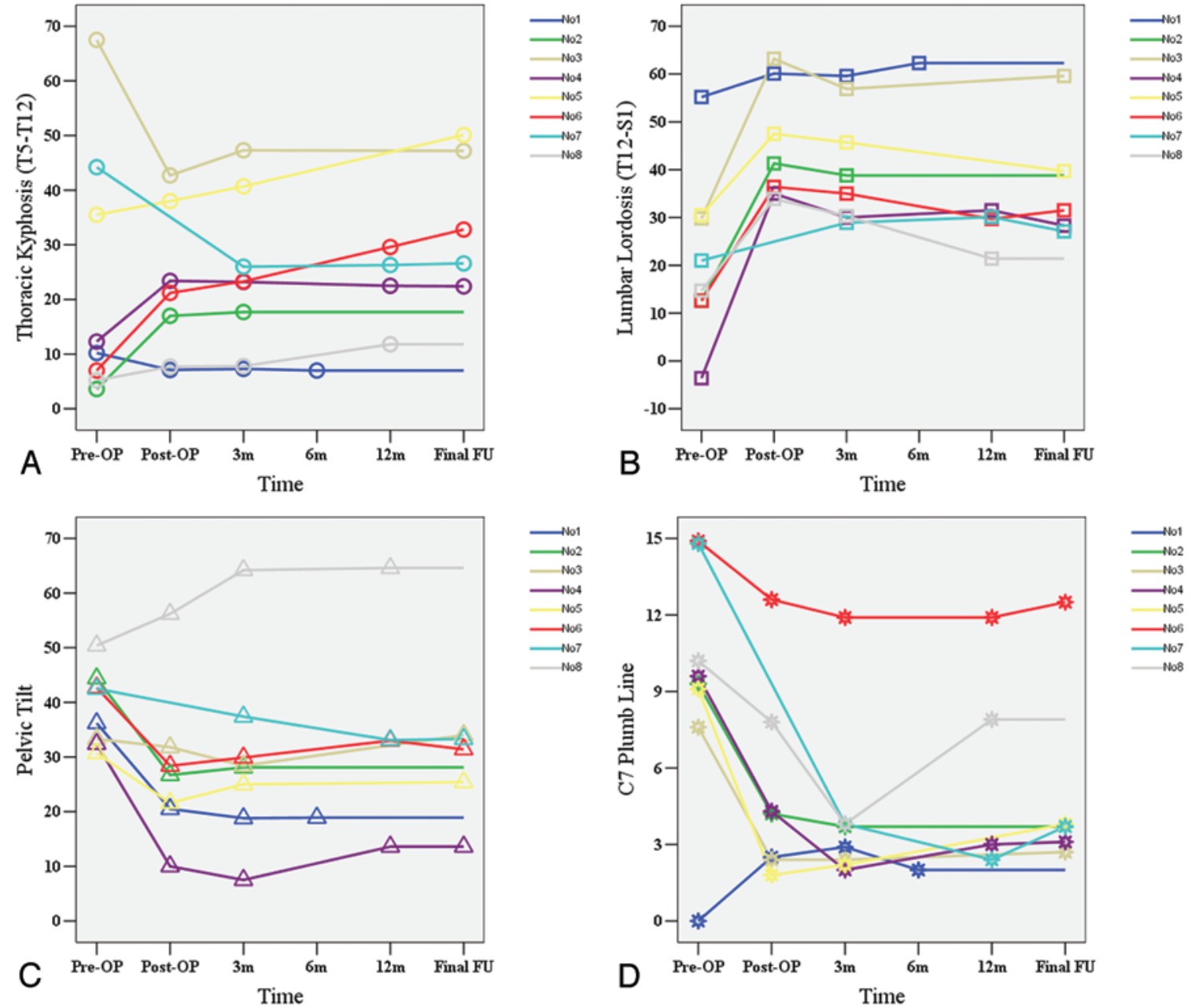

FIG. 3. Each of the lines on the graph represent a patient in the study $(n=8)$, and the markers (circle, square, triangle, and star) represent the radiological measurements (thoracic kyphosis, lumbar lordosis, pelvic tilt, and C-7 plumb line, respectively) preoperatively (Pre-OP), immediately postoperatively (Post-OP), at 3 months $(3 \mathrm{~m}), 6$ months $(6 \mathrm{~m})$, and 12 months $(12 \mathrm{~m})$ postoperatively, and at the final follow-up (Final FU). Figure is available in color online only.

had significant positive sagittal imbalance. All patients had undergone previous long fusion surgeries, extending back an average of 31 years (range 11-41 years). The demographic, surgical, and clinical outcome data of the patients are summarized in Tables 1 and 2.

\section{Perioperative Data}

The mean operative duration was $479.3 \pm 117$ minutes (range 327-620 minutes). The mean EBL was $1687.5 \pm$ $1287.8 \mathrm{ml}$ (range 300-4100 ml; Table 2). The perioperative complication rate was $25 \%$ (2/8 patients), which included persistent mild postoperative leg weakness in 1 patient (Case 2) and a small stitch abscess that resolved uneventfully in another (Case 4). Patient 7 did not have a perioperative complication but developed prominent implant and junctional cervicothoracic kyphosis 6 years postoperatively, which was treated with surgical revision.

\section{Radiological Outcomes}

Seven of 8 patients had a positive sagittal imbalance preoperatively; their mean C-7 plumb line was significantly improved from $10.8 \pm 2.9 \mathrm{~cm}$ (range $0-14.9 \mathrm{~cm}$ ) preoperatively to $5.3 \pm 3.6 \mathrm{~cm}$ (range $2-12.5 \mathrm{~cm}$ ) at the final follow-up $(t=4.864, p=0.003$; Fig. 3D). The mean lumbar lordosis in these patients at final follow-up was $35.2^{\circ} \pm$ $12.6^{\circ}$ (range $21.4^{\circ}-62.3^{\circ}$ ), which was significantly greater than the preoperative lumbar lordosis $\left(16.8^{\circ} \pm 11.8^{\circ}\right.$, range $12.6^{\circ}-51.2^{\circ}, \mathrm{t}=-4.366, \mathrm{p}=0.005$; Fig. 3B).

In general, thoracic kyphosis was stable postoperatively, although a mild angle change occurred in 2 patients $\left(12.1^{\circ}\right.$ in Case 5 and $11.6^{\circ}$ in Case 6$)$ over a 2 -year followup period (Fig. 3A). The median change in thoracic kyphosis between the first postoperative radiograph and the one obtained at the last follow-up evaluation was $2.6^{\circ}$ (interquartile range $0.6^{\circ}-9.8^{\circ}$; Fig. $3 \mathrm{~A}$ ). The mean pelvic tilt at final follow-up $\left(31.2^{\circ} \pm 15.3^{\circ}\right.$, range $\left.13.6^{\circ}-64.6^{\circ}\right)$ was reduced compared with preoperative mean pelvic tilt $\left(39.1^{\circ} \pm\right.$ $6.9^{\circ}$, range $30.7^{\circ}-50.4^{\circ}, t=2.019, p=0.083$; Fig. $3 C$ ). The mean change in the pelvic tilt between the first postoperative radiograph and the one at final follow-up was $3.5^{\circ} \pm$ $2.2^{\circ}\left(\right.$ range $\left.1.4^{\circ}-8.4^{\circ}\right)$. 
TABLE 1. Patient demographic data

\begin{tabular}{cccclcc}
\hline Case No. & Age $(\mathrm{yrs})$, Sex & BMI $\left(\mathrm{kg} / \mathrm{m}^{2}\right)$ & ASA Class & \multicolumn{1}{c}{ Diagnosis } & Yrs From Original Fusion & Follow-Up (mos) \\
\hline 1 & $53, \mathrm{~F}$ & 25.9 & 2 & Lumbar spondylosis & 32 & 6 \\
\hline 2 & $51, \mathrm{~F}$ & 21.5 & 1 & Flat back & 25 & 4 \\
\hline 3 & $66, \mathrm{~F}$ & 20.4 & 2 & Kyphoscoliosis & 41 & 24 \\
\hline 4 & $50, \mathrm{~F}$ & 34.3 & 2 & Flat back & 40 & 48 \\
\hline 5 & $54, \mathrm{~F}$ & 27.2 & 3 & Flat back & 32 & 30 \\
\hline 6 & $57, \mathrm{M}$ & 39.3 & 2 & Flat back & 31 & 27 \\
\hline 7 & $69, \mathrm{~F}$ & 19.8 & 2 & Flat back & 11 & 90 \\
\hline 8 & $50, \mathrm{~F}$ & 27.6 & 2 & Flat back & 33 & 12 \\
\hline
\end{tabular}

ASA = American Society of Anesthesiologists; $B M I=$ body mass index.

No hook loosening or displacement was observed in this case series. Case 7 developed prominence at the proximal end of the construct at 6 years postoperatively and then underwent surgical revision, but her hooks remained intact. Her radiological outcomes were evaluated just before the revision surgery (Fig. 3).

\section{Clinical Follow-Up Outcomes}

The clinical follow-up outcome of 6 patients was rated as favorable. Of those 6 patients, Case 7 initially improved but developed junctional kyphosis at 6 years postoperatively and then underwent a surgical revision. Her clinical outcome was evaluated at final follow-up after this revision surgery.

Patient 2 was rated "fair" because of the postoperative onset of left leg weakness, which did not resolve at final follow-up 4 months postoperatively. This leg weakness may have been due to the pedicle subtraction osteotomy (PSO) performed at L-3. Patient 6 was rated as having "unfavorable" outcome because his preoperative leg pain was not alleviated postoperatively.

\section{Discussion}

To the best of our knowledge, this is the first study that has described the multiple-hook technique to fixate a multilevel fusion mass in revision spinal deformity surgeries and evaluated its outcomes. Because of the small sample size and limited follow-up duration in the current study, the intent of this paper was not to introduce this method as a "must-do" technique but as a "could-be-done" technique that surgeons can resort to as an alternative. It is important to note that this technique only served to solve the problem of instrumentation, and the other steps of the operation were performed in their standard manner. The primary significance of this technique is that it makes use of the previous fusion mass - which was originally the obstacle for pedicle screw instrumentation-and uses it as an ideal basis whereby the multiple-hook construct can be readily applied. From our experience, it is an effective, reliable, and safe technique with little risk of blood loss and neural injury.

In general, the radiological evaluation demonstrated a durable effectiveness of this technique, as lumbar lordosis and spinal sagittal balance were well restored and maintained in most patients. Although Cases 6 and 8 had abnormal sagittal balances at final follow-up, this was due to a suboptimal deformity correction (L-3 PSO) and inadequate position at the time of radiography, respectively. Patient 7 developed cervicothoracic junctional kyphosis at 6 years postoperatively, but the hooks, which were located in the lumbar spine, remained intact. Although hooks have been shown to be less effective in curve correction and maintenance in spinal deformity surgery compared with pedicle screws, ${ }^{2,4}$ the hooks in the current study were not used to correct a deformity; instead, they assisted in maintaining sagittal correction in conjunction with the pedicle screws at other segments.

TABLE 2. Patient surgical data

\begin{tabular}{ccccllllll}
\hline $\begin{array}{c}\text { Case } \\
\text { No. }\end{array}$ & $\begin{array}{c}\text { Op Time } \\
\text { (mins) }\end{array}$ & $\begin{array}{c}\text { EBL } \\
(\mathrm{ml})\end{array}$ & $\begin{array}{c}\text { Previous } \\
\text { Fusion }\end{array}$ & Previous Implant & $\begin{array}{c}\text { Hook } \\
\text { Placement }\end{array}$ & $\begin{array}{c}\text { No. of } \\
\text { Hooks }\end{array}$ & $\begin{array}{c}\text { Fusion at } \\
\text { Current Surgery }\end{array}$ & $\begin{array}{c}\text { AP-Combined } \\
\text { Surgery }\end{array}$ \\
\hline 1 & 327 & 300 & T6-L4 & Harrington rod & T11-L3 & 2/side & T6-S1 & No & Yes \\
\hline 2 & 592 & 1000 & L1-5 & No & L1-3 & 3/side & T12-S1 & L-3 PSO & Yes \\
\hline 3 & 504 & 1300 & T5-L1 & No & T5-L1 & 6/side & T3-ilium & No & No \\
\hline 4 & 351 & 1200 & T7-L5 & Harrington rod & T9-11 & Lt 3, rt 4 & T4-ilium & T12/L1 L2/3 SPOs & No \\
\hline 5 & 374 & 2000 & T10-L4 & No & T10-L2 & 4/side & T9-L5 & L-3 PSO & Yes \\
\hline 6 & 620 & 4100 & T4-S1 & Pedicle screws (L3-S1) & T4-L2 & Lt 8, rt 7 & T4-ilium & L-3 PSO & No \\
\hline 7 & 589 & 3000 & T11-L5 & No & L1-5 & Lt 3, rt 4 & T4-ilium & T-12 PSO & Yes \\
\hline 8 & 477 & 600 & T3-L3 & Harrington rod & T9-L1 & 3/side & T7-ilium & No & Yes \\
\hline
\end{tabular}

$\mathrm{AP}=$ anterior-posterior; $\mathrm{SPO}=$ Smith-Petersen osteotomy.

* An average of 8.1 hooks (range 4-15 hooks) was used per patient. 
The rate of patients who had a favorable or fair clinical outcome at final follow-up was $87.5 \%$ (7/8 patients; 6 favorable, 1 fair). This was slightly lower than the improvement rate of $100 \%$ found in Noun et al.'s series of 10 flat-back cases with previous fusion treated with posterior closing-wedge vertebral osteotomy. ${ }^{6}$ However, 7 of their cases underwent pedicle screw instrumentation in the original surgery and thus did not bear the major revision difficulties encountered in our series.

The mean operative time (497 minutes) and blood loss $(1688 \mathrm{ml})$ in our series are comparable to those of Noun et al.'s revision flat-back series ${ }^{6}$ (210 minutes and $1800 \mathrm{ml}$ ) and Bridwell's series of 27 patients with fixed sagittal imbalance (744 minutes and $2396 \mathrm{ml}$ ) who had undergone a mean of 2.1 previous operations. ${ }^{1}$ These 2 reported case series are the most similar to ours in terms of diagnosis and surgical history, ${ }^{9}$ although the comparisons are of limited significance because they were reported in the early 2000 s and the levels of operation are unknown. In our series, the mean blood loss and the fact that only 3 patients had a blood loss greater than $1500 \mathrm{ml}$ are reasonably acceptable for such surgical settings. The rate of perioperative complications in our series was $25 \%(2 / 8)$, the same as in the series of Noun et al., ${ }^{6}$ and is comparable to the series of Bridwell et al. wherein 14 early complications occurred in 27 patients. ${ }^{1}$ Neither complication was related to the use of hooks. No dural tear occurred during hook placement site preparation. Additionally, no patient developed a cardiovascular or respiratory complication associated with the effects of substantial blood loss or operative time. In contrast, in O'Neill et al.'s series of 126 patients undergoing 3 -column osteotomy in which $96 \%$ were diagnosed with fixed sagittal imbalance, there were 8 deep venous thromboses, 2 pulmonary emboli, and 1 myocardial infarction found postoperatively. ${ }^{7}$

\section{Conclusions}

We have shown that a multiple-hook technique can be a useful and easy option for fixating a previous multilevel fusion mass in revision spinal deformity surgery. The biggest advantage of this technique is that it can be performed in a clinical setting whereas conventional instrumentation techniques are extremely difficult to apply and bear significant potential risks.

\section{References}

1. Bridwell KH, Lewis SJ, Lenke LG, Baldus C, Blanke K: Ped- icle subtraction osteotomy for the treatment of fixed sagittal imbalance. J Bone Joint Surg Am 85-A:454-463, 2003

2. Dobbs MB, Lenke LG, Kim YJ, Kamath G, Peelle MW, Bridwell KH: Selective posterior thoracic fusion for adolescent idiopathic scoliosis. Comparison of hooks versus pedicle screws. Spine (Phila Pa 1976) 31:2400-2404, 2006

3. Jang JS, Lee SH, Min JH, Maeng DH: Changes in sagittal alignment after restoration of lower lumbar lordosis in patients with degenerative flat back syndrome. J Neurosurg Spine 7:387-392, 2007

4. Kim YJ, Lenke LG, Cho SK, Bridwell KH, Sides B, Blanke $\mathrm{K}$ : Comparative analysis of pedicle screw versus hook instrumentation in posterior spinal fusion of adolescent idiopathic scoliosis. Spine (Phila Pa 1976) 29:2040-2048, 2004

5. Matsumoto M, Watanabe K, Tsuji T, Ishii K, Nakamura M, Chiba K, et al: Late instrumentation failure after total en bloc spondylectomy. J Neurosurg Spine 15:320-327, 2011

6. Noun Z, Lapresle P, Missenard G: Posterior lumbar osteotomy for flat back in adults. J Spinal Disord 14:311-316, 2001

7. O'Neill KR, Lenke LG, Bridwell KH, Hyun SJ, Neuman B, Dorward I, et al: Clinical and radiographic outcomes after 3-column osteotomies with 5-year follow-up. Spine (Phila Pa 1976) 39:424-432, 2014

8. Pheasant HC, Gilbert D, Goldfarb J, Herron L: The MMPI as a predictor of outcome in low-back surgery. Spine (Phila Pa 1976) 4:78-84, 1979

9. Wang Y, Boachie-Adjei O, Lenke LG (eds): Spinal Osteotomy. Dordrecht: Springer, 2015

10. Wiggins GC, Ondra SL, Shaffrey CI: Management of iatrogenic flat-back syndrome. Neurosurg Focus 15(3):E8, 2003

\section{Disclosures}

Dr. Wood has served as a consultant to AlphaTec Spine and K2M, and has received royalties from Globus Inc.

\section{Author Contributions}

Conception and design: Wood. Acquisition of data: both authors. Analysis and interpretation of data: Liu. Drafting the article: both authors. Critically revising the article: Wood. Reviewed submitted version of manuscript: Wood. Approved the final version of the manuscript on behalf of both authors: Wood. Statistical analysis: Liu. Study supervision: Wood.

\section{Correspondence}

Kirkham B. Wood, Department of Orthopaedic Surgery, Stanford University Medical Center, 450 Broadway St., M/C 6342, Pavilion C, 4th Fl., Redwood City, CA 94063-6342.email: kbwood@ stanford.edu. 